\title{
UNA MIRADA ECONÓMICA AL DISEÑO CONSTITUCIONAL CHILENO: IMPACTO SOBRE EL PROCESO LEGISLATIVO Y LA ACCIÓN DE LOS GRUPOS DE INTERÉS*
}

\author{
José Francisco García García \\ Sebastián Soto Velasco* * \\ "Caballeros, no confío en ustedes". \\ Gunning Bedford de Delaware \\ (Convención Federal de 1787) $^{1}$
}

\begin{abstract}
RESUMEN
El presente documento tiene por objeto analizar el proceso legislativo y el rol que cumple en éste la acción de los grupos de interés desde la perspectiva del análisis económico del derecho público. Para los autores es discutible que el único enfoque a la hora de abordar la acción de estos grupos sea la regulación especial, de las cuales la regulación del financiamiento de la política y del lobby son buenos ejemplos. En cambio, proponen mirar el diseño constitucional y el del proceso legislativo chileno, en particular las cortapisas que nuestra institucionalidad ha establecido para hacer más difícil el proceso de captura a los parlamentarios y más costoso conseguir aquellos acuerdos de mero beneficio especial o privado, o en donde no se considere el interés público o no se busque la provisión de bienes públicos. Así, se propone una teoría económica de la Constitución, que busca explicar la racionalidad de instituciones y mecanismos como el sistema bicameral, el veto presidencial, la delegación al Ejecutivo y la reserva legal, el respeto a las ideas matrices, la iniciativa exclusiva, la existencia de distintos tipos de quorums, el control jurídico de la constitucionalidad de las leyes por parte del Tribunal Constitucional, entre otros.
\end{abstract}

Trabajo recibido el 22 de abril de 2009, y aprobado el 6 de mayo del mismo año.

** El primero es abogado. Licenciado en Derecho, PUC. (LL.M) Master y Doctorando en Derecho (JSD), Universidad de Chicago. Profesor e Investigador Facultad de Derecho, Universidad del Desarrollo. Correo electrónico: jfgarcia@udd.cl. El segundo es abogado. Licenciado en Derecho y profesor Facultad de Derecho, PUC. (LL.M) Master en Derecho, Universidad de Columbia. Director del Programa Legislativo de Libertad y Desarrollo. Correo electrónico: ssoto@lyd.org Buchanan, James M. y Gordon Tullock, The Calculus of Consent: Logical Foundations of Constitutional Democracy. University of Michigan Press, Ann Arbor, 1962. 


\begin{abstract}
The paper develops an economic theory of the Chilean Constitution and the legislative process, and the role that play interest groups in the later. For the authors is not clear that the only approach when analyzing interest group politics consists, inevitably, in establishing statutory regulations, like campaign finance laws and lobbying regulation. To the contrary, this paper suggests that the Chilean Constitution and the design of the legislative process should be considered more seriously. Different constitutional principles, institutions and mechanisms are established in the Chilean constitutional design that raises the costs of capturing the State and Congress. Thus, the authors propose an economic theory of the Chilean constitution and analyze different topics such as the bicameral system, executive veto, delegation of legislative powers to the Executive and the legal reserve clause, the respect for the matrix ideas of a legislative proposal, quorums, judicial review of legislation by the Constitutional Court, among others.
\end{abstract}

PALABRAS CLAVES

Derecho constitucional, análisis económico del derecho, constitución, proceso legislativo.

\title{
KEY WORDS
}

Constitucional law, law and economics, constitution, legislative process.

\section{INTRODUCCIÓN}

La acción de los grupos de interés y la vinculación que éstos tienen con legisladores y autoridades públicas es un tema que ha motivado diversas respuestas en Chile y en el mundo. En nuestro país, algunas de ellas son leyes vigentes desde hace años ${ }^{2}$ y otras están a un paso de $\operatorname{serlo}^{3}$. Éstas y otras regulaciones similares suelen ser consideradas por diversos sectores de la sociedad como medidas fundamentales para el perfeccionamiento de la democracia y, desde una mirada más profunda aunque mal enfocada desde nuestra perspectiva, antídoto contra la "excesiva" influencia de los grupos de interés en el proceso político.

Sin embargo, no puede desconocerse la existencia de grupos que buscan influenciar las

Ley 19.884, sobre Transparencia, Límite y Control del Gasto Electoral.” En: http://www.bcn.cl [visitado el 06.01.09].

"Proyecto de Ley, $\mathrm{N}^{\circ}$ Boletín: $3407-7$ [regula el lobby]". En: http://sil.congreso.cl/pags/index.html [visitado el 06.01.09]; y “Proyecto de Ley, $\mathrm{N}^{\circ}$ Boletín: 6189-6 [Establece normas sobre la actividad de lobby.]". En: http:/ /sil.congreso.cl/pags/index.html [visitado el 06.01.09]. 
Una mirada económica al diseño constitucional chileno: impacto sobre el proceso legislativo y la acción de los grupos de interés.

decisiones de la autoridad. De hecho, el conflicto de fondo, esto es, la lucha de diversos intereses, está en el corazón mismo de un sistema democrático. ¿Puede pensarse la política en forma independiente de los intereses? ¿Puede una democracia existir sin grupos de interés tratando de influir en el debate público? ¿Es posible la competencia política sin conflictos de intereses? Estas preguntas envuelven en sí resoluciones legislativas que están conectadas a definiciones sobre derechos y distintas concepciones de la política.

El presente documento pretende avanzar en algunas respuestas a estas preguntas. En primer lugar, busca avanzar en el camino de introducir la mirada del análisis económico del derecho público o la aplicación del Public Choice o Teoría de la Elección Pública al derecho ${ }^{4}$; para analizar el diseño constitucional y la potencial acción de los grupos de interés en el proceso legislativo. El uso de este enfoque basado en el análisis económico de las reglas e instituciones legales -su racionalidad, eficiencia e incentivos en los individuos e instituciones públicas- es una nueva mirada que el mundo del derecho público chileno está recién empezando a conocer ${ }^{5}$.

Una segunda aspiración de este documento dice relación con discutir acerca de la mejor manera de abordar la influencia de los grupos de interés en el proceso legislativo. Como se verá, en nuestra Constitución y proceso legislativo encontramos mecanismos que buscan encausar la acción de los grupos de interés y hacer más costoso el proceso de captura de los parlamentarios y de la regulación. En efecto, nuestro diseño institucional genera algún grado de incertidumbre en el proceso de captura ${ }^{6}$ del Congreso al contemplar un sistema bicameral,

4 Para ver un análisis introductorio de este tópico respecto del proceso político y legislativo ver García, José Francisco. 2008b. "El Proceso Legislativo sin Romance", Ius et Praxis Año 14 №2.

Autores del mundo del derecho público que han tocado algunos aspectos de esta Escuela o se han referido tangencialmente a esta: Barandarián, Edgardo. 2001. "La regulación de los derechos fundamentales", Revista Chilena de Derecho Año 28, No2, pp. 363-394; Montt, Santiago. 2004. "Balmaceda y la Nitrate Railways Company: La primera gran controversia regulatoria en Chile y su impacto en la Guerra Civil de 1891", Revista de Derecho Administrativo Económico, Año 13, pp. 65-98; Fermandois, Arturo, Derecho Constitucional Económico: Garantías Económicas, Doctrina y Jurisprudencia. Ed. Universidad Católica, Santiago, 2006, $2^{\circ}$ ed., p. 333; Delaveau, Rodrigo. 2006. "La regulación expropiatoria en la jurisprudencia norteamericana", Revista Chilena de Derecho, Año 33, No3, pp. 411-432; Soto, Sebastián. 2007. "Las Comisiones Mixtas en las Reformas Constitucionales: Efectos de la Reforma al Artículo 127 de la Constitución” XXXVII Jornadas de Derecho Público. Universidad Católica de Valparaíso. (inédito); García, José Francisco. 2008a. "Modelos de regulación del lobby en el derecho comparado", Revista Chilena de Derecho, Año 35, pp. 107-134; Romero, Juan José, "Cambio en la regulación eléctrica y estabilidad en los contratos: ¿Cuándo una limitación se transforma en privación? ¿Cuándo compensar?”, en Sentencias Destacadas 2007, (Ed.) Arturo Fermandois, Libertad y Desarrollo, Santiago, 2008, pp.19-60; y, Silva Méndez, Ernesto. 2008. "Introducción a la Economía Política Constitucional”, Actualidad Jurídica (Universidad del Desarrollo), №18, pp. 189-204.

6 Por captura, que será utilizada en relación al Estado, Congreso o parlamentarios indistintamente, entendemos los distintos esfuerzos de grupos de interés particular (e.g., empresarios, sindicatos, colegios profesionales, gremios, etc.) por obtener del Estado a través de diferentes vías lícitas o ilícitas (e.g., lobby, tráfico de influencia, financiamiento de campañas electorales, extorsión, etc.) diversos beneficios (e.g., regulaciones, subsidios, transferencias, barreras a la entrada a competidores, etc.) que tienen un claro interés particular (a dicho grupo), y que la mayoría de las veces, se opondrá al bienestar social. 
un fuerte rol del Ejecutivo en el proceso legislativo, reglas supermayoritarias en algunos ámbitos, posibilidad de delegación restringida, exigencia de respetar las ideas matrices, etc. Todo esto no impide la captura, pero la hace más costosa. Por eso lo relevante será detenerse en algunas instituciones y analizarlas desde esta perspectiva.

Para esto, se analizará el diseño constitucional y legislativo de nuestro país enfocándose en los mayores costos de transacción (y captura) que éste genera, tanto desde el punto de vista de los grupos de interés como de los parlamentarios. De este modo, se pretende hacer un aporte al análisis de instituciones de nuestra democracia desde la perspectiva de la teoría de la elección pública y el derecho, para así contribuir al estudio de la acción de los grupos de interés en Chile.

\section{UNA MIRADA ECONÓMICA AL DISEÑO CONSTITUCIONAL CHILENO}

Habiendo dedicado algunas páginas al estudio más teórico del análisis económico del derecho público o aplicación del public choice al derecho y su enfoque analítico frente al proceso legislativo -y qué podemos esperar de la influencia de los grupos de interés- es momento de detenerse en su aplicación y consecuencias para el diseño constitucional del país. Asimismo corresponde también analizar el modo en que diversas instituciones operan como verdaderas cortapisas al accionar de los grupos de interés y a los acuerdos de claros beneficios privados que pudieran acordarse en el Congreso.

Si bien en Chile el análisis económico del derecho constitucional no ha sido un tema de gran investigación académica, nuestro diseño institucional cuenta con variados mecanismos de la acción de los grupos de interés en el mercado político. Por eso, a continuación, nos detendremos en algunas instituciones y reglas que se insertan dentro del procedimiento legislativo y permiten vislumbrar que, en la práctica legislativa, tanto la presión que pueden ejercer estos grupos como los beneficios que pueden ofrecer los legisladores se encuentran limitados.

\subsection{Sistema Bicameral}

Uno de los aportes más importantes de El Cálculo del Consenso ${ }^{7}$, fue su apoyo explícito a un modelo bicameral para la conformación de una legislatura. Este análisis nos lleva a la teoría de las "coaliciones mínimas ganadoras"8 de Ricker quien sostiene que, en teoría, la coalición más estable en un cuerpo legislativo estará compuesta por una mayoría levemente superior al cincuenta por ciento (mayoría simple). Una coalición de mayor tamaño tratará de

Buchanan, James M. y Tullock, Gordon, The Calculus, cit. nota n.1.

Ricker, William H., The Theory of Political Coalitions. Yale University Press, New Haven, 1962. 
Una mirada económica al diseño constitucional chileno: impacto sobre el proceso legislativo y la acción de los grupos de interés.

sacarse de encima el "exceso de mayoría" como medio para mejorar los pagos per capita a los miembros de la coalición.

Buchanan y Tullock, sin embargo, ofrecieron un análisis un tanto más problemático respecto del tamaño efectivo de una coalición ganadora (gobernante). Los autores demostraron que con una regla de mayoría simple en una legislatura unicameral, un cuarto del electorado puede formar una coalición electoral efectiva9 .

El análisis de Buchanan y Tullock respecto del bicameralismo, descansa sobre la base de los beneficios asociados con aumentar el cálculo efectivo del consenso entre los electores que tienen el poder de controlar la dirección de la legislación en una legislatura unicameral. Si en cambio tenemos una legislatura bicameral, y cada cámara responde a mapas geográficos diseñados de manera diferente, luego el efecto es aumentar el tamaño de la coalición electoral ganadora potencial desde poco más de un cuarto a poco más de 7/16, o casi la mitad ${ }^{10}$. Para alcanzar el mismo nivel de consenso usando una legislatura unicameral, se necesitaría reemplazar la regla de la mayoría simple con una regla supermayoritaria de 7/8. La dificultad de usar una regla como esta para descabezar a una coalición electoral ganadora tan pequeña consiste en que la regla alternativa permitiría que 1/16 del electorado tuviera un poder de veto efectivo sobre la legislación propuesta. En efecto, el bicameralismo aumenta el consenso a poco más de la mitad sin entregar un poder de veto a una pequeña minoría del electorado ${ }^{11}$.

Para Levmore, una mejor explicación para el bicameralismo se encuentra en que aumenta el tamaño de la coalición electoral gobernante mientras permite a su vez lo que él denomina "ganadores condorcet fuertes" ${ }^{12}$, refiriéndose a aquella propuesta que prevalecerá en cada

Considere un cuerpo legislativo donde cada congresista representa un distrito geográfico. Por simplicidad, asumamos que existen 100 distritos de aproximadamente el mismo tamaño y población, cada uno con un solo representante. Buchanan y Tullock demostraron que debido a que una mayoría simple de legisladores pueden controlar la mayoría de los resultados en el Congreso y porque una mayoría simple de electores en los distritos de los congresistas son quienes controlan el que sean electos, el resultado final es que con un $26 \%$ (el producto de un medio de los legisladores por un medio de los electores de estos) del electorado de un cuerpo unicameral se forma una coalición electoral que controle el proceso legislativo.

10 Buchanan, James M. y Tullock, Gordon, The Calculus, cit. nota n. 1, pp. 242-244.

11 Stearns, Maxwell L. y Zywicki, Todd, Public Choice concepts and Applications in Law. (Inédito, ver. 2008) c.4, p. 33.

12 Levmore, Saul. 1992. "Bicameralism: When Are Two Decisions Better Than One?", International Review of Law and Economics, $\mathrm{N}^{\circ} 12$, pp. 145-198. El ganador condorcet, es lo contrario que sucede en los "ciclos" que se analizan bajo "la paradoja de la votación", uno de los casos más interesantes de la Teoría de la Elección Social, sub-disciplina estrechamente relacionada von la Teoría de la Elección Pública. Esta última puede ilustrarse con tres elecciones efectuadas por tres votantes sinceros (no-estratégicos), cuyas preferencias individuales se encuentran ordenadas numéricamente y son transitivas (es decir, que cuando una persona prefiere A sobre B y B sobre $\mathrm{C}$, del mismo modo prefiere A sobre $\mathrm{C}$ ). Ello implica que no hay un mecanismo de votación que prevenga que se genere un ciclo entre las opciones cuando se votan de a pares. Así, cualquier opción que derrote a otra será a su turno derrotada cuando sea enfrentada contra una tercera. El resultado dependerá enteramente en el orden en el cual las opciones sean presentadas. Así, la alternativa condorcet es la que le gana al resto de las 
cámara en un determinado tópico. Adicionalmente, el autor sugiere que el bicameralismo aumenta los costos a los controladores de agenda (agenda setters) en cada cámara dado que, para que se apruebe cualquier proyecto de ley, debe ser aprobado por ambas cámaras con el mismo texto. Finalmente, para Levmore resolver el problema del consenso en una legislatura unicameral requeriría de establecer una regla de supermayoría. El criterio de condorcet está basado en una norma mayoritaria, y así la dificultad con una regla de votación supermayoritaria es que hace más complicado la habilidad de la legislatura para asegurar ganadores condorcet fuertes -aquellos que sobreviven en ambas cámaras- en el proceso legislativo.

En Chile la discusión en torno al bicameralismo no ha sido un tema particularmente álgido. De hecho, ya la Constitución de 1822 estableció un Congreso Bicameral y, desde esa fecha, las constituciones siguientes han contemplado la existencia de un Congreso de dos cámaras ${ }^{13}$. De este modo, nuestra institucionalidad ha respondido desde los inicios de su vida independiente a un modelo que dificulta la captura de los grupos de interés y aumenta el consenso necesario para aprobar las leyes.

Adicionalmente el sistema de representación en el Congreso se funda tanto en el territorio como en la población. De este modo, el Senado tiene una representación territorial y así cada región está representada por dos senadores o, excepcionalmente, cuatro. La Cámara de Diputados, en cambio, distribuye sus cupos sobre la base de la población y por eso la Región Metropolitana elige a 32 diputados y la XI Región de Aisén a solo 2. En este sentido, los grupos de interés deben promover legislaciones que consideren esta diversidad en la representación. Una iniciativa que se construya sobre un regionalismo excesivo puede superar el Senado pero avanzar con mayor dificultad en la Cámara de Diputados ${ }^{14}$.

\subsection{Logrolling, ideas matrices e iniciativa exclusiva}

\subsubsection{Logrolling o intercambio de votos}

El logrolling es la posibilidad de intercambiar o "transar" votos entre parlamentarios. Mercuro y Medema explican de manera simple este concepto. "En el proceso de logrolling, el

opciones. Así, el sistema político debe estar diseñado para generar ganadores condorcet y evitar por un lado perdedores condorcet (la alternativa que siempre pierde frente a las demás cuando compiten de a pares) y ciclos. Entre otras cuestiones un sistema bi-partidista facilita romper el problema de los ciclos, ya que elimina uno de los requistos para los ciclos: la existencia de al menos tres electores.

13 Carrasco, Sergio, Génesis y Vigencia de los Textos Constitucionales Chilenos. Editorial Jurídica, Chile, 2002, p. 58.

14 El proyecto de ley de Casinos - actual ley 19.995- es un ejemplo. La disposición que prohibía la existencia de casinos en la Región Metropolitana fue discutida largamente en la Cámara de Diputados e incluso amenazó la aprobación de todo el proyecto. En el Senado, en cambio, la norma no despertó el mismo interés. Ver Sesión 70 de la Cámara de Diputados, 29 de abril de 2003. 
Una mirada económica al diseño constitucional chileno: impacto sobre el proceso legislativo y la acción de los grupos de interés.

representante A accede a votar por un proyecto de ley que el representante B quiere que sea aprobado, y en cambio B vota por la legislación que interesa a A. Los legisladores que están en minoría respecto a un tema en que están altamente interesados, pueden, a través del logrolling, estar dispuestos a intercambiar sus votos en otros temas en los cuales ellos no se encuentran tan interesados a cambio de los votos de otros legisladores y, en el proceso, construir una coalición de mayoría a favor de sus temas preferidos. Desde la perspectiva de la eficiencia, el logrolling es una espada de doble filo: permite propuestas eficientes que de otro modo no tendrían mayoría; sin embargo, del mismo modo, permite la aprobación de propuestas ineficientes que de otro modo no se adoptarían" 15 . Este fenómeno se produce como vía para superar el problema de la incapacidad de los votantes para manifestar la intensidad de sus preferencias, en un contexto donde no existe un mercado para comprar y vender votos ${ }^{16}$.

Esta visión del proceso legislativo como una red de intercambios es similar en algunos aspectos a un mercado privado pero con importantes calificaciones. Una de las más importantes es que los intercambios en el mercado privado dependen generalmente del consentimiento unánime de las partes a intercambiar y se presumen son generadoras de aumentos de bienestar o pareto-superiores. En cambio, el proceso legislativo ocurre a través de variadas reglas de mayoría o súper mayorías pero rara vez, de unanimidad. Como resultado, para Stearns y Zywicki, "incluso si las leyes resultantes cumplen con la eficiencia de Kaldor Hicks, es decir, implicando que en el total se aumenta el bienestar, los ganadores y perdedores de todos modos se mantienen" 17 .

En este contexto, para algunos el logrolling se considera lo opuesto al "bien público" y a un proceso legislativo y de toma de decisiones deliberativo, ya que permite a los grupos de interés presionar para que se legisle directamente a favor de sus intereses especiales o se adjunten beneficios privados a la legislación de interés público. Sin embargo, si la política es de hecho un proceso de intercambios entonces puede que no haya nada intrínsecamente malo con el intercambio de votos. Aún las leyes que persiguen el interés público a menudo tendrán efectos distributivos desiguales y, si bien puede haber aumentos de bienestar, es inevitable que ciertas

15 Mercuro, Nicholas y Medema, Steven G, Economics and the Law. Princeton University Press, New Jersey, 2006 2ed, p.176.

16 Para Levmore, Saul. 2000. "Voting Whit Intensity”, Stanford Law Review, No53, pp. 111-145: "los mercados convencionales permiten que las preferencias intensas sean satisfechas ya que los participantes pueden demandar, o sustituir por, cosas que ellos quieren. De hecho existen pocas áreas donde las preferencias intensas no puedan ser satisfechas. En la mayoría de estas áreas, sin embargo, la riqueza da poder a los jugadores para satisfacer sus preferencias, y esto presenta un problema cuando el derecho al voto está en juego, ya que estos derechos son a menudo ejercidos precisamente donde hemos decidido tomar decisiones a través de políticas más que de mercados. La pregunta obvia en el caso del derecho a votar es si las preferencias intensas pueden ser acomodadas sin transferir un enorme poder a los ciudadanos acaudalados".

17 Stearns, Maxwell L. y Zywicki, Todd, Public, cit. nota n. 11, p. 40. 
José Francisco García García

Sebastián Soto Velasco

personas estén en desventaja incluso por una ley benigna o de interés general. En este contexto, el logrolling podría ser simplemente un forma de pagos laterales en compensación a los "perdedores" tras la adopción de una determinada ley que emana del superávit de mayor bienestar social creado por la nueva ley. Así como los pagos compensatorios a veces van envueltos en intercambios pareto superiores -por ejemplo compensar a terceros que sufren externalidades negativas en un contrato privado-, del mismo modo, estos pagos compensatorios pueden surgir en los procesos políticos. Alternativamente, el logrolling puede verse como el medio a través del cual los grupos de interés obtienen ventajas a través del proceso político a cambio de permitir que una determinada legislación sea aprobada ${ }^{18}$.

En este escenario, la teoría del public choice revela que, sin logrolling, aquellos que tienen preferencias menos marcadas -midiendo éstas según las ganancias o pérdidas esperadas- son normalmente aquellos que dominan la votación. En cambio, la posibilidad de negociar los votos permite que los representantes puedan transar sus preferencias más débiles a cambio de obtener apoyo en aquellas que son más intensas.

Finalmente, para el Public Choice es previsible observar otras dos consecuencias del fenómeno del logrolling. La primera es que se puede anticipar que aumentarán los proyectos de ley aprobados y, en especial, aquellas iniciativas que crean franquicias tributarias, aranceles o tarifas ${ }^{19}$. La consecuencia lógica de esto es un aumento en el gasto público y un crecimiento del aparato estatal. La segunda crítica supone que, como es natural, los proyectos aprobados serán financiados con impuestos. De este modo, el logrolling no solo permite que se aprueben iniciativas que normalmente (o siempre) son ineficientes y aumentan el gasto público, sino que también permite que los favorecidos con la iniciativa distribuyan en otros los costos asociados.

\subsubsection{El diseño institucional chileno frente al logrolling}

El diseño institucional de nuestro país ofrece dos instituciones que en cierta medida limitan la transacción de votos en el proceso de formación de la ley. Estas son el mandato constitucional que impone que las indicaciones a los proyectos de ley deben respetar sus ideas matrices y las materias de iniciativa exclusiva del Presidente de la República.

18 Como sostienen Stearns y Zywicki: "En política, así como en el fútbol americano, es generalmente más fácil bloquear que dar un pase. Aquellos grupos que por diversos motivos tienen el poder de evitar la aprobación de una determinada legislación deseada, podrían usar el logrolling simplemente como un medio para obtener beneficios legislativos incluso cuando todo el paquete legislativo que se está negociando no imponga costos exclusivamente sobre ellos”. Stearns, Maxwell L. y Zywicki, Todd, Public cit. nota n.11, p. 40.

19 La literatura de Estados Unidos también menciona como ejemplo paradigmático de iniciativas que emanan de la posibilidad de logrolling el así llamado "pork barrel". Esto es, proyectos de ley que contienen numerosas iniciativas y programas con financiamiento público que son aprobados y financiados gracias a la negociación de los votos. 
Una mirada económica al diseño constitucional chileno: impacto sobre el proceso legislativo y la acción de los grupos de interés.

\section{a) Ideas matrices en los Proyectos de Ley}

El respeto a las ideas matrices durante la discusión legislativa se incorporó a la Constitución de 1925 a fines del gobierno del Presidente Frei Montalva. Posteriormente la Constitución de 1980 reiteró la norma prohibiendo, en el artículo 69, la discusión de indicaciones, esto es, adiciones o correcciones que realicen los parlamentarios o el Presidente de la República a un proyecto de ley, si éstas no tienen relación directa con las ideas matrices o fundamentales de la iniciativa que está siendo tramitada ${ }^{20}$.

Esta norma limita el logrolling pues lo circunscribe a materias que deben tener alguna vinculación con la idea matriz del proyecto de ley en discusión. De este modo, un legislador no podrá transar su voto a cambio de la aprobación de una indicación completamente ajena a la idea fundamental de la iniciativa. Aún cuando el logrolling puede subsistir si se trata de dos iniciativas contenidas en proyectos de ley distintos, lo cierto es que es más costoso hacer exigible el acuerdo en la tramitación de proyectos que no son votados al mismo tiempo.

Ya el Presidente Frei Montalva, refiriéndose en 1970 a la reforma que incorporó esta materia a la Carta Fundamental, advertía que la norma pondría "fin al mal hábito de las llamadas 'leyes misceláneas', que tratan de toda clase de asuntos, que se prestan al abuso frecuente de que se aproveche una ley importante para introducirle disposiciones destinadas a favorecer a determinados grupos y aún personas individuales" ${ }^{21}$.

La incorporación de la norma constitucional vino a limitar estas prácticas tan habituales en el pasado. Desde los noventa, la doctrina y el Tribunal Constitucional han tenido ocasión de analizar el tema en numerosas ocasiones. El Tribunal, por ejemplo, ha profundizado sus alcances y entregado nuevos criterios de interpretación con el ánimo de dar certeza al momento de interpretar la disposición e iluminar su aplicación en la discusión legislativa ${ }^{22}$.

Todo esto ha creado una verdadera jurisprudencia en virtud de la cual hoy existe un marco jurídico que impide discutir en los proyectos de ley asuntos ajenos a las ideas fundamentales del mismo. De este modo, la transacción al interior del Congreso se reduce considerablemente.

Al respecto ver el artículo 23, 24 y 32 de la Ley Orgánica Constitucional del Congreso Nacional. Ver también STC Rol No 91, c. 2 que, conociendo del control preventivo de la LOC del Congreso, declaró inconstitucional la posibilidad de presentar "indicaciones tendientes a la mejor resolución del asunto" en la Cámara o el Senado.

21 Discurso del Presidente Frei sobre Reformas Constitucionales. 30 de diciembre de 1969. Piedrabuena, Guillermo, La Reforma Constitucional. Historia de la Ley 17.284, Sus Principales Alcances y Posición de los Partidos Políticos. Editorial Eros, santiago, 1970, p. 168.

22 Ver STC Rol N ${ }^{\circ}$ 410, 413 y 786. Ver también Buchheister, Axel y Soto, Sebastián, "Ideas Matrices en los Proyectos de Ley: Inconsistencias del Tribunal Constitucional”, en Sentencias Destacadas 2004. (Ed.) Fermandois, Arturo, Libertad y Desarrollo, Santiago, 2005a. 
José Francisco García García

Sebastián Soto Velasco

En efecto, por un lado disminuye la presión de los grupos de interés pues éstos solo podrán promover nuevas regulaciones cuando estén en discusión proyectos de ley cuya idea matriz tiene relación con sus intereses. Además también disminuyen los temas que pueden ser transados por los parlamentarios al momento de decidir su voto. La norma constitucional exige que esas materias que serán transadas se limiten a temas que tengan una relación directa con la idea matriz o fundamental del proyecto de ley en tramitación.

\section{b) Materias de Iniciativa Exclusiva del Presidente de la República}

Las materias de iniciativa exclusiva del Presidente de la República son aquellas que solo pueden discutirse cuando el Presidente de la República lo determina ${ }^{23}$. Recae sobre esa autoridad la decisión de presentar proyectos de ley o indicaciones sobre las materias que la Constitución califica como de su iniciativa exclusiva. Como veremos, esta es otra forma de reducir el logrolling en el proceso legislativo.

Variadas fueron las justificaciones que se utilizaron para limitar la iniciativa parlamentaria. Una de ellas se funda en la acción de los grupos de interés. Como en su momento expuso Sergio Molina, refiriéndose a la reforma de 1970 que limitó la iniciativa parlamentaria, "lo que sucede es que la presión o presencia de ciertos grupos induce a tomar iniciativas en su favor, sin ponderar debidamente todas las repercusiones que esto puede acarrear. (...) Es difícil para un parlamentario -concluye- negarse a este tipo de demandas porque (...) constituyen grupos con fuerza electoral y política"24.

Hoy las normas sobre seguridad social, aquellas que conceden beneficios pecuniarios y otras tantas vinculadas con áreas comúnmente citadas por la doctrina como bienes transables en el mercado político -tales como exenciones, tributos, etc.- son de iniciativa exclusiva del Presidente de la República. Esto ha conducido a una disminución del campo de temas que pueden ser objeto de transacción al momento de votar pues un legislador, para ofrecer una regulación A incluida dentro de aquellas de iniciativa exclusiva a cambio del voto favorable en otra regulación $\mathrm{B}$, requerirá el patrocinio del ejecutivo incorporando un nuevo actor al proceso de logrolling. Adicionalmente, también se limita el campo de influencia de los grupos de interés y la gama de ofertas que pueden poner a disposición de éstos los legisladores.

23 Hoy, por ejemplo, se cuentan dentro de estas materias aquellas que tienen relación con la alteración de la división política o administrativa del país o con la administración financiera o presupuestaria del Estado; las que imponen, supriman, reduzcan o condonen tributos; las que creen nuevos servicios públicos o empleos rentados, sean fiscales o semifiscales; las que estén dirigidas a contratar empréstitos o celebrar cualquier otra clase de operación que comprometa el crédito o la responsabilidad financiera del Estado; las que fijen, modifiquen o aumenten remuneraciones y pensiones; las que establezcan las modalidades y procedimientos de negociación colectiva; y las que determinen las normas sobre seguridad social. Ver Art. 65 de la Constitución.

24 Frei, Eduardo; Molina Sergio; Evans, Enrique; Cumplido, Francisco; Silva, Alejandro y Lagos, Gustavo, La Reforma Constitucional de 1970. Editorial Jurídica, Chile, 1970, p.81. 


\subsection{Quórum: entre la unanimidad y la regla de mayoría}

Para Buchanan y Tullock, en la obra tantas veces citada ${ }^{25}$, el diseño constitucional es un asunto que consiste en último término determinar qué regla de votación o mecanismo de elección sería especificado por la Constitución para legitimar la actividad del Estado. En este sentido, una opción a la hora de buscar decidir acerca de decisiones colectivas -y un punto de partido analítico-, consiste en adoptar la regla de la unanimidad ${ }^{26}$. El atractivo de esta regla dice relación con que es la única que asegura que las modificaciones legales sean cambios hacia un estado donde todos los miembros de la comunidad queden en mejor posición; si producto del paquete legislativo, regla o nueva regulación uno de estos miembros queda en una situación peor, esta persona puede votar en contra y rechazar el cambio legal. Aquellos que abogan por el uso de esta regla suelen basar su argumentación en la creencia de que un Estado que actúa sobre la base del consenso voluntario y unánime, es por definición neutral, no coercitivo y por tanto legítimo.

Sin perjuicio de sus propiedades atractivas desde el punto de vista de la eficiencia paretiana, la regla de unanimidad tiene dos importantes dificultades asociadas a ella. Primero, es muy costoso, en términos de tiempo y otros recursos, diseñar una propuesta que generará unanimidad, sobre todo considerando que los individuos tienen distintos gustos y preferencias. En segundo lugar, al requerir la unanimidad, se entrega a los individuos un incentivo para actuar de manera estratégica u oportunista (hold out), amenazando con vetar la legislación propuesta a menos que se le entreguen beneficios adicionales o se le reduzcan los costos de adoptar dicha legislación. Todos estos costos podrían perfectamente exceder los beneficios obtenidos con la legislación misma. Dados estos y otros obstáculos, es poco probable que se aprueben nuevas reglas o leyes, o se modifiquen las actualmente existentes, precisamente por la adopción del criterio de la unanimidad. Puesto de otra forma, al poner en manos de un solo individuo el poder de veto, la unanimidad tiende a perpetuar el status quo.

Si la unanimidad es problemática, entonces la cuestión a resolver es cuál es la regla óptima de votación para la sociedad. En general la respuesta obvia es la regla de mayoría. Sin embargo, la regla de mayoría no garantiza que sólo las leyes, reglas o regulaciones que sean pareto superiores (que no dejen a nadie en una situación peor y al menos beneficien a uno) sean adoptadas. También esta regla permite que una mayoría redistribuya los recursos sociales escasos desde la minoría para si misma. Los orígenes teóricos de este análisis también pueden ser encontrados en el trabajo de Wicksell, quien reconoció que una regla de votación de mayoría simple resultaría en una mayoría aprobando leyes que concentraran los beneficios para ellos 
mientras establecieran aumentos de impuestos (costos) en una minoría o distribuyéndolo entre todos en cantidades muy pequeñas -donde los beneficiarios constituyen sólo una parte-. Esto ha llevado a algunos críticos a cuestionar la legitimidad de la legislación mayoritaria sobre la base de que las leyes pasadas por una regla de mayoría simple son inconsistentes con el Estado de Derecho $^{27}$.

Así las cosas, al considerar que todas las reglas de votación son costosas, la elección pública busca identificar aquellas reglas que generaran resultados políticos eficientes. Buchanan y Tullock comienzan definiendo los costos asociados con diversas reglas de votación, centrándose en la suma de (i) los costos externos de la toma de decisiones (EC) -los costos que sufren aquellos que desaprueban una decisión- y (ii) los costos de toma de decisión (DM) -los costos de negociación asociados a la toma de una decisión-. Del Gráfico1 se puede observar que EC disminuye mientras el número de votantes requeridos para aprobar un proyecto $(\mathrm{N})$ aumenta, dado que son cada vez menores los costos que podemos imponer en terceros cuando vamos tomando decisiones bajo reglas que se van acercando cada vez más a la unanimidad. Obviamente, la unanimidad sería la regla eficiente si DM fuera cero, al minimizar EC. DM, sin embargo, es una función creciente de $\mathrm{N}$, dado que se hace crecientemente más costoso traer a más y más personas al proceso de toma de decisión mientras consideramos adoptar reglas de decisión que se acerquen a la unanimidad. La suma de los costos externos y los costos de toma de decisión reflejan el costo total de imponer cualquier regla particular de consentimiento. La magnitud percibida de DM es tal que incluso aquellos que están más predispuestos a la unanimidad -incluidos Wicksell, Buchanan y Tullock- han argumentado que la eficiencia requiere de una regla de votación algo menor a la unanimidad.

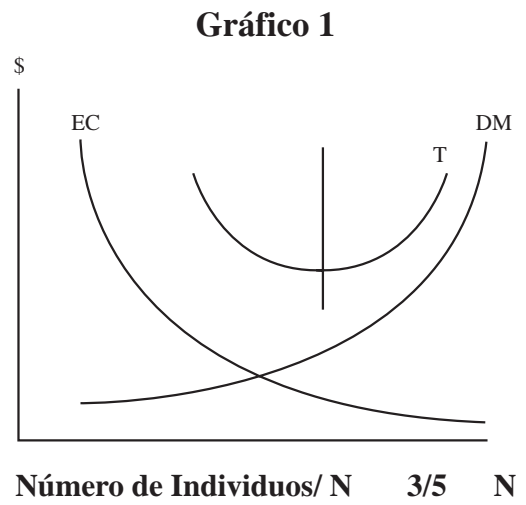

27 Halper, Louise A. 1993. "Parables of Exchange: Foundations of Public Choice Theory and the Market Formalism of James Buchanan", Cornell Journal of Law and Public Policy, N², pp. 231-233- 
Una mirada económica al diseño constitucional chileno: impacto sobre el proceso legislativo y la acción de los grupos de interés.

La meta desde la perspectiva de la eficiencia, entonces, es determinar la regla de votación que minimiza la suma de estos costos. Esto ocurre en los 3/5 del Gráfico 1. No esperamos, sin embargo, que la regla de mayoría que minimice los costos sea la misma para diferentes tópicos, dado que EC y DM tienden a variar para cada tema ${ }^{28}$. La tarea en la etapa o período constitucional (constituyente) -donde son determinadas las reglas del juego- consiste, entonces, en decidir, para cada categoría de política pública, la regla de decisión que minimiza la suma de estos dos costos analizados. La distribución óptima de votos requerida para adoptar una propuesta legislativa ocurre en el punto más bajo de la curva de costos TC. Al alejarse de la regla de unanimidad y elegir la regla de mayoría "óptima", la sociedad, en efecto, elige la regla más eficiente, tomando en consideración todos los costos envueltos en el proceso de votación ${ }^{29}$. Es en este sentido que una Constitución "racional" o diseñada "económicamente" permitirá la adopción de decisiones colectivas específicas que no cumplan con el criterio de Pareto. Esto implica que se tomarán decisiones que no cumplan con la regla de la unanimidad, sin embargo, cuando son miradas desde una perspectiva más amplia -el período constitucional- estas decisiones colectivas logran obtener un status de legitimidad. Ello ocurre porque, aunque la decisión no sea pareto-eficiente (en este caso requiriendo $3 / 5$ en vez de la unanimidad), la decisión habrá sido tomada basada en una regla que, a priori, minimiza la suma de los costos externos y de los costos de toma de decisión a la sociedad y por tanto tiene la virtud de ser minimizadora de costos.

En Chile el diseño constitucional y legislativo se ha hecho cargo de la discusión respecto sobre las mayorías óptimas para tomar decisiones colectivas. En efecto, si bien la regla de la unanimidad no es común, en el proceso de formación de la ley pueden encontrarse ese tipo de exigencias para modificaciones del procedimiento de tramitación de la ley. Así por ejemplo, tanto el artículo 24 del Reglamento de la Cámara de Diputados como el artículo 17 del Reglamento del Senado disponen que la unanimidad de los comités o de los miembros presentes pueden suspender la aplicación de una disposición reglamentaria para un asunto concreto. Asimismo, también son los reglamentos los que exigen la unanimidad para proceder en ciertos casos. De este modo, cuando los diputados forman la Tabla de Despacho Inmediato se requiere el acuerdo unánime de los jefes de comité ${ }^{30}$ y cuando se quiere omitir que un proyecto se dirija a una Comisión -salvo en el caso de la de Hacienda- se requiere la unanimidad de la sala ${ }^{31}$. En el Senado, la unanimidad de la Sala o de los Comités puede omitir el primer informe de una comisión ${ }^{32}$.

28 La idea de que una determinada regla de votación no es optima para cada tema es desarrollada por Mueller, Dennis, Public Choice III, Cambridge University Press, Cambridge, 2003, pp. 74-76.

Buchanan, James M. y Gordon Tullock, The Calculus, cit. nota n. 1, pp. 43-84.

Artículo 100 del Reglamento de la Cámara de Diputados.

Artículo 118 del reglamento de la Cámara de Diputados.

Artículo 36 del Reglamento del Senado. 
José Francisco García García

Sebastián Soto Velasco

En estos casos, la unanimidad pareciera estar justificada pues los casos en los que se pide el acuerdo unánime dicen relación con modificaciones en las reglas del juego del proceso legislativo. Si la mayoría, o aún una mayoría calificada, pudieran modificar dicho proceso sin contar con el consentimiento de todos los participantes, se incentivaría la eliminación de trámites legislativos con los que contaba la minoría que no conciente. Por lo demás, la unanimidad no aumenta considerablemente los costos pues lo único que impide no obtenerla es modificar para un caso concreto el reglamento que regula la tramitación de la ley. Si no se obtiene se deberán seguir los trámites regulares.

Por otra parte, el Congreso también conoce en el proceso legislativo otros quórum distintos de la unanimidad. Como se sabe, la Constitución de 1980 incorporó a nuestra institucionalidad una serie de normas que, para su aprobación requieren del voto favorable de un número de diputados y senadores que representen más de la mayoría simple de los integrantes de ambas cámaras. Son éstas las normas de quórum calificado, orgánico constitucionales e interpretativas de la Constitución. Para aprobar las primeras se requiere de la concurrencia de la mayoría absoluta de los diputados y senadores en ejercicio. Las normas de rango orgánico constitucional requieren del voto favorable de $4 / 7$ de los diputados y senadores en ejercicio y las interpretativas de la Constitución, de $3 / 5^{33}$.

En términos generales, las normas que requieren de un quórum especial son aquellas que se refieren a materias fundamentales que regulan instituciones relevantes en un sistema democrático. Aún cuando pudiera discutirse esta última afirmación y cuestionar el rango de algunas de las materias que son objeto de normas de quórum calificado u orgánico constitucional, lo cierto es que instituciones percibidas como esenciales en un Estado de Derecho requieren para su modificación de la concurrencia de algo más que una simple mayoría. En efecto, las funciones esenciales del Congreso Nacional, del Ministerio Público, de la Contraloría General de la República y del Tribunal Constitucional, entre tantos otros, requieren para su aprobación del voto favorable de 4/7 de los diputados y senadores en ejercicio. El mismo quórum se requiere para determinar la organización básica de la Administración del Estado y la organización y atribuciones de los tribunales de justicia. Curiosamente, la creación de impuestos se realiza por medio de normas de rango simple.

Más importante aún es el quórum de 2/3 que contempla la Constitución para modificar los capítulos donde se estatuyen las bases y principios de protección de las libertades y derechos individuales, es decir, el capítulo I -bases de la institucionalidad- y el III -derechos y garantías constitucionales-. Esta justificación pretende hacer más difícil que la democracia o el juego de las mayorías circunstanciales puedan redistribuir derechos de la minoría.

33. Artículo 66 de la Constitución. 
Una mirada económica al diseño constitucional chileno: impacto sobre el proceso legislativo y la acción de los grupos de interés.

Por último cabe destacar que la jurisprudencia del Tribunal Constitucional y la práctica legislativa han ido ampliando o restringiendo, según el caso, el ámbito de las normas de quórum especial. Para ello ha generado una serie de criterios iluminan el debate legislativo al momento de calificar el quórum de una norma ${ }^{34}$. Es interesante señalar que, el único dato que conocemos, calculado para la Cámara de Diputados y considerando únicamente los proyectos de ley que contenían normas de rango orgánico constitucional, nos muestra que aproximadamente el 35\% de los proyectos de ley discutidos en la legislatura ordinaria de los años 2003 y 2004 contenían normas de rango orgánico constitucional ${ }^{35}$.

\subsection{Delegación y Reserva Legal}

La delegación de potestades reguladoras que efectúa el Poder Legislativo en el Ejecutivo suele justificarse por diversas razones, tanto prácticas como políticas. Siguiendo a Stearns, entre las primeras encontramos la intención de reducir el trabajo del Congreso, evitar reformas legales cada vez que cambian las circunstancias, permitir que los especialistas decidan las regulaciones de materias donde los congresistas no tienen conocimiento suficiente y facilitar respuestas rápidas. Desde un punto de vista político, la justificación más recurrente de la delegación en el poder ejecutivo es la intención de "despolitizar" la toma de decisiones.

Con todo, la teoría del public choice nos presenta otras razones. El mismo Stearns manifiesta que los legisladores pueden usar la delegación para dejar que todos los grupos de interés clamen victoria en el proceso legislativo (beneficios), mientras culpan a la agencia del Ejecutivo en un tiempo futuro por imponer los $\operatorname{costos}^{36}$. En la misma línea, Aranson, Gelhorn y Robinson argumentan que "los legisladores, en promedio, preferirían apostar por delegaciones regulatorias cuando se enfrentan con electores con preferencias intensas y conflictivas, más que por no proveer legislación o por proveer legislación específica que resuelva los intereses en conflicto. Si los legisladores proveen legislaciones específicas a favor de un grupo, inevitablemente comprometen el apoyo de al menos otro de los grupos en conflicto. Si, al contrario, ellos deciden no legislar, ellos aparecerían como no interesados o empáticos frente a los problemas o cuestiones de dichos grupos, comprometiendo potencialmente el apoyo de ambos grupos" ${ }^{37}$.

Como hemos visto anteriormente, el modelo transaccional explica que la delegación a una autoridad del Ejecutivo ocurrirá en presencia de costos concentrados y beneficios

34 Buchheister, Axel y Soto, Sebastián. 2005b. “Criterios para la Calificación de las Normas Orgánico Constitucionales en la Jurisprudencia del Tribunal Constitucional”, Revista Chilena de Derecho, Vol. 32 N².

35 Buchheister, Axel y Soto, Sebastián. "Criterios", cit. nota n. 34, p. 258.

36 Stearns, Maxwell L. 1992. "The Public Choice Case Against the Item Veto", Washington and Lee Law Review, No 49, pp. 397.

37 Aranson, Peter H., Gelhorn, Ernerst y Robinson, Glen O. 1982. "A Theory of legislative Delegation”, Cornell Law Review, № 68, pp. 1-34 
José Francisco García García

Sebastián Soto Velasco

distribuidos, pero con fuerza en legislaciones en que tantos los beneficios como los costos son concentrados. Esto, como se dijo, se debe a que los intereses en conflicto alcanzan gran intensidad en ambos lados de la ecuación: por una parte quienes pueden obtener una legislación favorable y por la otra quienes soportaran el gravamen para financiarla.

En Chile la delegación que puede efectuar el Congreso Nacional en la administración tiene limitaciones más rigurosas que las existentes, por ejemplo, en Estados Unidos. En este último país la práctica constitucional, manifestada en la llamada non delegation doctrine, exige que las leyes delegatorias contengan un "principio inteligible" en virtud del cual las agencias puedan guiar su acción regulatoria. Más allá de ello, lo cierto es que desde 1935 la Corte Suprema americana no ha invalidado ninguna ley en virtud de esta doctrina. Este contexto facilita la delegación a las agencias e incentiva la presión de los grupos de interés sobre la burocracia $^{38}$.

En nuestro país el asunto es más complejo. Sabemos que la Constitución reserva a la ley la regulación de ciertas materias que no pueden, en consecuencia, ser reglamentadas por el Presidente de la República en el ejercicio de su potestad reglamentaria de ejecución. Es lo que se denomina el principio de reserva legal en cuyos alcances y perspectivas no hay mayores acuerdos ni en la doctrina ni en la jurisprudencia ${ }^{39}$. Con todo, y más allá de las incertidumbres, lo cierto es que el marco constitucional limita, en algún grado, la posibilidad de delegar en la administración la regulación de ciertos temas. Los legisladores tienen que asumir costos ante los grupos de interés y regular en la ley materias que, eventualmente, les sería más atractivo delegar en la administración ${ }^{40}$.

38 Heinzerling, Lisa y Tushnet Mark, The Regulatory and Administrative State, Materials, Cases, Comments. Oxford University Press, 2006, pp. 665-682.

39 Por todos, ver Fermandois, Arturo, Derecho, cit. nota n. 5, p. 333. y García, Gonzalo, La Reserva Legal de Derechos Constitucionales: ¿Poder Legislativo contra la Administración?. Ediciones Universidad Alberto Hurtado, 2004. En el Tribunal Constitucional, por ejemplo, en materia de regulación económica se encuentran sentencias que van desde un reconocimiento a la reserva legal absoluta, como Publicidad Caminera I (STC Rol $\mathrm{N}^{\circ}$ 146, de 21 de abril de 1992) y II (STC Rol N 167, de 6 de abril de 1993), y Ley de Bases del Medio Ambiente (STC Rol No 185 de 28 de febrero de 1994), a una relativización extrema de la reserva legal, como el caso Catalíticos (STC Rol No 325 de 26 de junio de 2001).

40 Un ejemplo reciente lo constituye la tramitación de una modificación a la ley de impuesto territorial, Ley 20.033 de 1 de julio de 2007. El texto presentado por el gobierno en la Cámara de Diputados y discutido en la sala de la misma corporación contenía una norma que delegaba en la administración la determinación de la tasa correspondiente al impuesto territorial que se aplica a los bienes raíces. De este modo, el proyecto discutido en la Cámara de Diputados señalaba que las respectivas tasas "se deberán fijar (por el Servicio de Impuestos Internos) en un rango entre el 5 y el 15 por mil" (Informe Complementario de la Comisión de Gobierno Interior. 07/08/2003. Boletín 2892-06). Como explicó en su momento el Diputado Paya "esta disposición faculta a la autoridad administrativa para determinar múltiples tasas tributarias. El proyecto no señala cuántas tasas serán, ni su monto, ni los tramos de avalúo a los que se van a aplicar" (Sesión de la Cámara de Diputados N 28, de 12 de agosto de 2003). Como en su momento se argumentó, delegar en la administración la determinación de las tasas del impuesto territorial dentro de un rango autorizado en la ley, como buscaba la indicación, no sería 


\subsection{El veto presidencial}

En la literatura de la elección pública, el veto presidencial tiende a operar como un actor relevante en el proceso legislativo. Por un lado, permite que el Ejecutivo sea una de las partes negociadoras y, en la misma línea, exige que para obtener la aprobación de una ley el Ejecutivo no esté en contra. Este escenario tiene efectos fundamentales desde el punto de vista de los grupos de interés pues aumentan los costos de transacción para llegar a un acuerdo. En efecto, ya no basta contar con los votos de dos ramas del Congreso sino que se debe incluir un nuevo actor que cuenta con dinámicas, incentivos y una agenda distinta. En la misma línea, el veto fragiliza y genera incertidumbre respecto del acuerdo alcanzado en el Congreso.

La existencia del veto no debe llevarnos a pensar que el Presidente actuará únicamente pensando en el interés general del país. Como es de suponer, el veto también sirve para derribar una legislación de interés general que genera daño o costos a quien ocupa el Poder Ejecutivo. A contrario sensu, efectivamente el Presidente tiene un arma poderosa para poder derribar los acuerdos de mero interés especial que han sido generados entre parlamentarios y grupos de interés, cuando crea que está en juego el interés general de la nación.

En Chile, el Poder Ejecutivo participa en el proceso de formación de la ley con importantes atribuciones. Así por ejemplo puede iniciar la tramitación de proyectos de ley, presentar indicaciones y manejar la agenda legislativa a través de las urgencia. A ello debe agregarse la posibilidad que tiene el Presidente de la República de formular observaciones a los proyectos aprobados por el Congreso Nacional ${ }^{41}$.

El llamado derecho a veto puede ser ejercido por el Presidente manifestando la desaprobación total con el proyecto o su desacuerdo parcial. En la práctica puede reemplazar alguna disposición aprobada por el Congreso (veto sustitutivo), eliminarla (veto supresivo) o incorporar una nueva materia (veto aditivo). Enfrentadas a un veto, ambas cámaras pueden aprobarlo sin introducirle modificaciones o rechazarlo. En este último caso, el Congreso puede insistir con su propuesta para lo que necesitará del voto favorable de $2 / 3$ de los diputados y senadores en ejercicio. Si, en cambio, rechaza el veto del Ejecutivo y una o las dos cámaras no alcanzan el quórum para insistir no hay ley respecto de los puntos en discrepancia ${ }^{42}$.

admisible en virtud del principio de reserva legal en materias tributarias. Más tarde en el Senado se aprobó una indicación para fijar las tasas del impuesto en la ley. De este modo, se logra reducir la presión de los grupos de interés - por ejemplo los municipios - sobre la administración pues ésta deja de fijar discrecionalmente la tasa. Es el Congreso Nacional el que lo hace circunscribiendo, al menos para este caso, la influencia de los grupos de interés nada más que al proceso legislativo.

41 Artículo 73 de la Constitución y Título III de la Ley Orgánica Constitucional del Congreso Nacional.

42 Artículo 36, LOC Congreso Nacional. 
José Francisco García García

Sebastián Soto Velasco

\subsection{Interpretación judicial y rol del TC}

¿Es necesario un mayor control judicial respecto de la legislación especial que obtendrían los grupos de interés?

Para algunos este mayor control es necesario porque que el proceso político tiende a sobre-beneficiar los intereses particulares de determinados grupos de interés en perjuicio del interés social ${ }^{43}$. En este sentido, algunos autores, sostienen que los jueces deberían emplear herramientas de interpretación (estándares o criterios de interpretación judicial) para hacer más difícil la captura por parte de los grupos de interés ${ }^{44}$.

Para otros académicos, este debate se debe abordar desde la perspectiva de la legislación antimonopolio vehículo apropiado para el control judicial de la "captura" de la regulación por parte de los grupos de interés ${ }^{45}$.

De todos modos, todas estas posiciones de mayor intervención o revisión judicial han sido fuertemente criticadas tanto porque existe poca evidencia empírica que determine cuanta "captura" existe en realidad en el proceso político, como porque no es claro que el proceso judicial sea más fácil de capturar que el proceso político ${ }^{46}$.

En nuestro país el Tribunal Constitucional pasa a ser un nuevo actor en el proceso de

43 Chemerinsky, Erwin. 1989. "The Supreme Court, 1988 Term-Foreword: The Vanishing Constitution", Harvard Law Review, No103, pp. 43-87; Epstein, Richard A. 1984. "Toward a Revitalization of the Contract Clause", University of Chicago Law Review, No51 pp. 703-745, y, Takings: Private Property and the Power of Eminent Domain. Harvard University Press, Cambridge, 1985, p. 306; Mashaw, Jerry L. 1980. "Constitutional Deregulation: Notes Toward a Public, Public Law”, Tulane Law Review, N54, pp. 849-896, y 1989. "The Economics of Politics and the Understanding of Public Law," Chicago Kent Law Review, N65, pp. 123; Sunstein, Cass R. 1984. "Naked Preferences and the Constitution", Columbia Law Review, No84, pp.1689-1714, y, 1985. "Interest Groups in American Public Law", Stanford Law Review, N³8, pp. 29-67.

44 Sunstein, Cass R. 1989 "Interpreting Statutes in the Regulatory State”, Harvard Law Review, No103, pp. 405451; Macey, Jonathan R. 1986. "Promoting Public Regarding Legislation Through Statutory Interpretation: An Interest Group Model”, Columbia Law Review, No86, pp. 223-256; Eskridge Jr., William N. 1988. "Politics Without Romance: Implications of Public Choice Theory for Statutory Interpretation", Virginia Law Review, $\mathrm{N}^{0} 74$, pp. 275-310.

45 Wiley Jr., John S. 1986. “A Capture Teory of Antitrust Federalism”, Harvard Law Review, N99, pp. 713-745; Robinson, Glen O. 1988. "Public Choice Speculations on the Item Veto", Virginia Law Review, No 74, pp. 408456.

$46 \quad$ Véase generalmente a Elhauge, Einer 1991. "Does Interest Group Theory Justify More Intrusive Judicial Review?", Yale Law Journal, №101, pp. 31-75, donde argumenta que los grupos de interés podrían no ser tan influyentes en el proceso político, y que las propuestas para expandir el control judicial sufren de la falacia de nirvana, ya que el proceso de adjudicación, como el de regulación, son imperfectos y susceptibles a la influencia de los grupos de interés. También Farber, Daniel A. y Frickey, Philip P. 1987. "The Jurisprudence of Public Choice", Texas Law Review, N65, p. 873. 
Una mirada económica al diseño constitucional chileno: impacto sobre el proceso legislativo y la acción de los grupos de interés.

formación de ley, al menos respecto de aquellas leyes en que la Constitución exige el control preventivo de constitucionalidad. En concreto, nuestra Constitución dispone que aquellos proyectos que contengan normas de rango orgánico constitucional o interpretativas de la Constitución están sometidos al control automático por parte del Tribunal. Adicionalmente, un cuarto de los integrantes del Senado o de la Cámara de Diputados pueden requerir al Tribunal Constitucional su pronunciamiento respecto de una ley o proyecto de ley que consideren inconstitucional $^{47}$.

Como puede apreciarse, la presión de los grupos de interés, además de las dos cámaras del Congreso y el Presidente de la República, también debe considerar el rol que el Tribunal Constitucional puede jugar en la reducción (o disuasión) del papel de los grupos de interés. En este contexto, cabe preguntarse si es posible que el TC chileno juegue un mayor rol al ejercer el control preventivo de legislación especial. Consideramos que más allá de elaborar criterios para definir cuándo estamos frente a una legislación de claro interés privado y cuándo de interés público -cuestión de por sí bastante discutible- se hace necesario que el TC establezca por la vía jurisprudencial definiciones claras de conceptos como bien común, interés general, utilidad pública o discriminación arbitraria (conceptos jurídicos indeterminados). Al mismo tiempo, es recomendable que el TC empiece a elaborar ciertos estándares que permitan una aproximación más cierta al análisis constitucional que realiza. De esta forma será posible dilucidar en qué circunstancias se augura un escrutinio más riguroso de un proyecto o de una ley y en cuales el TC será más deferente al trabajo del Congreso ${ }^{48}$. Ello podría depender, entre otras cosas, en si el proyecto aprobado tiene o no carácter de legislación especial.

De este modo, desarrollando una metodología de análisis para enfrentar ciertas regulaciones que afectan derechos individuales se envía una poderosa señal para el legislador respecto de los beneficios o gravámenes tolerables que puede imponer a los individuos o grupos de individuos.

\section{CONCLUSIONES Y PROYECCIONES}

El presente trabajo busca mostrar los incentivos que afectan la conducta de actores racionales. Por supuesto, el análisis económico no pretende analizar todos y cada uno de los aspectos que están en juego a la hora de analizar el diseño constitucional y el proceso legislativo,

$47 \quad$ Artículo 93, № 1 y 3, Constitución Política de la República.

48 La jurisprudencia de la Corte Suprema de Estados Unidos nos ofrece numerosos de estos test que han sido desarrollados en casos emblemáticos. Por mencionar algunos, Lemon test (para fallar causas en las que está en juego la norma constitucional que prohíbe aprobar una ley que adopte una religión como la oficial del Estado); Penn Central Test (para determinar casos de regulación expropiatoria); New York Times v. Sullivan (establece cuando un funcionario público puede demandar daños por críticas falsas vinculadas a su conducta oficial); Chevron (para analizar la interpretación de las leyes realizadas por las agencias gubernamentales); etc. 
pero ha demostrado ser capaz de entregar herramientas y metodologías útiles. Esta aproximación nos ayudará a entender con mayores instrumentos analíticos las fuerzas que hay, por ejemplo, tras la búsqueda de votos (logrolling) para aprobar el financiamiento del Transantiago o por qué ciertas industrias o sectores productivos, sindicatos o gremios buscan obtener legislación que les entregue subsidios, franquicias tributarias o impongan barreras a la entrada a la competencia.

Por supuesto, este enfoque ha recibido críticas, tanto por académicos del mundo de las ciencias sociales, como del derecho público ${ }^{49}$. Básicamente se cuestiona asuntos relativos a la evidencia empírica de sus postulados; su enfoque metodológico; sus implicancias normativas conservadoras; su visión pesimista respecto de la democracia; y, sus implicancias desde la perspectiva jurídica.

Esperamos también que este documento sea considerado como un punto de partida para futuras investigaciones y desarrollos posteriores de este enfoque hacia otras áreas del derecho público. En particular, y aún cuando el grueso de este análisis se ha realizado sólo respecto del proceso legislativo, entendemos que buena parte de la captura del Estado y sus regulaciones se produce más bien ante las agencias gubernamentales o en los gobiernos locales, áreas en que queda mucho por decir. 29-67; Kelman, Mark 1988. "On Democracy-Bashing: A Skeptical Look at the Theoretical and "Empirical" Practice of the Public Choice Movement", Virginia Law Review, No74, pp. 199-234; y Farber, Daniel A. y Frickey, Philip P. 1987 "The Jurisprudence”, cit. nota n. 46, pp. 873-905. Como respuesta a este artículo véase a DeBow, Michael y Lee, Dwight. 1988. "Understanding (and Misunderstanding) Public Choice: A Response to Farber and Frickey”, Texas Law Review, №66, pp. 93-112. 